e. Maintaining a overview of the total program in order to put into better perspective the balance between the magnitude of effort and the quality of the output for programs and sub-projects,

f. Utilizing experience or knowledge in the fields of research reactor design, accelerator design, magnet systems, energy storage and transfer systems, etc.

It should be expected that the results from these design efforts will then form a basis for a more realistic scheduling of effort and distribution of funding.

The evaluation of the development of the Group III-S and two of the Group II type of facilities and their significance to the concept of neutron sources are to be a phase of the overall design effort.

The auxiliary heating aspects of the Group II-S type concepts are identified in the tables. Converting these into neutron source concepts must necessarily involve some magnetic confinement technique of either the linear theta-pinch or the mirror type system. Therefore, the concepts can be categorized as Group III type facilities.

\title{
G. Remarks
}

The CTR programmatic needs for materials radiation studies are for 14 $\operatorname{MeV}(D, T)$ neutrons at flux levels of approximately $10^{14} \mathrm{n} / \mathrm{cm}^{2}-\mathrm{sec}$. Depending on the specific objectives of each program, the neutron energy spectra requirements may range from a sharply defined peaked distribution at $14 \mathrm{MeV}$, to a variety of spectra from $14 \mathrm{MeV}$ on-down which are characteristic of the neutronic environment anticipated in fusion reactors. Ideally it is advantageous to develop a neutron source facility with a sharply peaked distribution at $14 \mathrm{MeV}$ and design-in a "spectrum-tailoring" capability.

As previously mentioned other systems may be utilized as neutron generators. The class of accelerators and light-element target systems, such as Be, $\mathrm{Cu}$, etc., already exist and do not require further development by the CTR program. There are many accelerators (1inacs and cyclotrons) with beam energies ranging from 20 to $50 \mathrm{MeV}$ and beam currents of $10-150 \mu \mathrm{A}$ and Be target systems which yield neutron fluxes in the range of $>10^{13} \mathrm{n} / \mathrm{cm}^{2}-\mathrm{sec}$. 
A sensitive measure of merit in assessing accelerator and light-element target systems is the strongly angular-dependent neutron-energy spectrum. For light-element targets the average eners; of the emitted neutron is about 0.4 of the incident deuteron energy, with the average energy increasing for the lower $Z$ elements. For deuteron energies greater than $5 \mathrm{MeV}$ and thick target systerns, the neutron spectra will be broad and ranging continuously from the eV region to energies several tens of $\mathrm{MeV}$ greater than the incident deuteron energy. For example, the upper range of the neutron distribution lies in the $20 \mathrm{MeV}$ region for $16 \mathrm{MeV}$ incident deuterons.

The emitted neutrons for high incident deuteron energies are strongly forward-biased with the energy-spectrum broadening becoming more pronounced at increasing deuteron energies (20-50 MeV) and at angles away from the forward direction. The energy half-width for $40 \mathrm{MeV}$ deuterons on Be is about 20 $\mathrm{MeV}$ at the average energy of $14.5 \mathrm{MeV}$.

Therefore, the use of these neutron sources must be based on the users ' ar.d reactor designers' evaluation that the output of the facility is the quality output needed to make technically significant advances directly relevant to the CTR program and to do this in the most economical manner. 


\section{A REPORT ON THE DEVELOPMENT OF INTENSE NEUTRON SOURCE FACILITIES \\ FOR THE}

CTR TECHNOLOGY PROGRAM

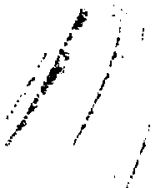

Including

A Summary of the Meeting

on the

Fusion-Neutron Source Development AEC-Germantown

December 5, 1972

\section{P. J. Persiani}

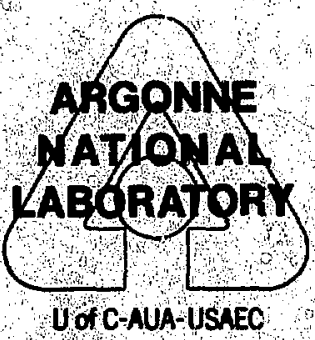

August 1973 
The facilities of Argonne National Laboratory are owned by the United States Govern ment. Under the terms of a contraet (W-31-109-Eng-38) between the U. S. Atomic Energy Commission, Argonne Universities Association and The University of Chicago, the University employs the staff and operates the Laboratory in accordance with policies and programs formulated, approved and reviewed by the Association.

\section{MEMBERS OF ARGONNE UNIVERSITIES ASSOCIATION}

The University of Arizona Carnegie-Mellon University

Case Western Reserve University The University of Chicago

Univerdity of Cincinnati Illinois Institute of $T$ echnology University of Illinois Indiana University Iowa State University The University of Iowa
Kansas State University The University of Kanes Loyola University Marquette University Michigan State University The University of Michigan University of Minnesota Univer bity of Mis souri Northwestern University University of Notre Dame
The Ohio Sta te Unive rsity Oho University

The Pennsylvania state University Purdue University

Saint Louis Univer bity

Southern Ilinois Univer ity The University of Texas at Austin Washington University Wayne State University The University of wisconsin

\section{NOTICE}

This report was prepared as an account of work sponsored by the United States Government, Neither the United States nor the United States Atomic Energy Commission, nor any of their employees, nor any of their contractors, subcontractors, or their employees, makes any warranty, express or implied, or as sume any legal liability or responsibility for the accuracy, completenes s or usefulnes $s$ of any information, apparatus, product or process disclosed, or represents that it s use would not infringe privately-owned rights.

\section{$+4$}




\title{
A REPORT ON THE DEVELOPMENT
}

\section{OF INTENSE NEUTRON SOURCE FACILITIES}

\author{
FOR THE
}

CTR TECHNOLOGY PROGRAM

\author{
Including
}

A Summary of the Meeting

on the

Fusion-Neutron Source Development

AEC-Germantown

December 5, 1972

NOTICE

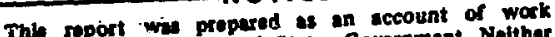
Thi soport wh propared ste Gavernment. Nother sonsored by the nor the United States Atomic Enory the United States not the United states Alonet not any of Comminaton; nor sny of thet omployen, nor anyes,

their contrectors; subcontractore, or thor employes,

makes eny wrranty, expreas or ingthe, or anum, com-

ievel liabihty or respontiblity for the eccuracy, com-

plotenes of uefulnes of any information, epparatus,

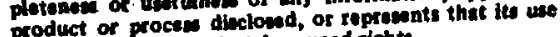

product of procese privitely owned rights.

P. J. Persiani

Argonne National Laboratory 
Table of Contents

Page No.

I. Surmary of Meeting on the Fusion-Neutron Source

Deve lopment held at AEC-Germantown, December 5, 1972

II. The Development of Intense Neutron Source Facilities 8 for the CTR Technology Program

A. Introduction 8

B. Classification of $(D, T)$ Neutron Facilities 8

C. Design-Related Considerations 10

D. Major Research and Development Areas 14

D.1 Introduction 14

D.2 Subsystem Development Areas Common to Several Types 14

a. Radiation Effects on Materials 14

b. Pulsed Energy Storage and Transfer System 15

E. Further Consideration on Source Facilities 24

E.1 Thermonuc lear-Radiation Facility 24

(Group III and Group III-S)

E.2 Group II and Group II-S Concepts 25

E.3 Target Development (Group I) 26

F. Sumary 26

G. Remarks 29 
I. Summary of Meeting on the Fusion-Neutron Source Development Held at AEC-Germantown, December 5, 1972

The participants were:
R. F. Post, LLL
R. L. Morse, LASL
R. Emigh, LASL
G. Miley, U of Illinois
A. Chilton, $U$ of Illinois
P. J. Persiani, ANL
J. Purcel1, ANL
A. Hertzberg, $U$ of Washington
G. VIases, U of Washington
F. Tenny, Princeton University
W. L. Rice, DCTR AEC

W. C. Gough, DCTR AEC

F. Tesche, DCTR AEC

W. G. Davey, U of Texas, Austin

O. E. Morgan, ORNL

A. Robson, NRL

J. Luce, LLL

S. D. Putnam, PI

T. Onkawa, GGA

R. E. Aamodt, U of Colorado

T. Kammash, $U$ of Michigan

G. Sawyer, LASL

The objective of the meeting was to explore the potential for developing fusion devices into plasma-driven neutron sources.

A survey and assessment of neutron source facilities for satisfying the needs of the CTR technology program has been mainly concerned with: a) the facilities currently available at AEC laboratories and institutions; and b) the concepts for source development as proposed by investigators in these organizations. The survey and evaluation is the result of the active participation by the materials science and neutron source researchers from Gulf General Atomics, MIT, University of Illinois, University of Wisconsin, Livermore, Los Alamos, Oak Ridge and Argonne.

An area that has not been considered is that of generating neutrons with a fusion power device. Several suggestions have been made to explore the potentials for the near-term (10 to 15 years) development of a materials and engineering test reactor based on the mirror and linear theta-pinch principle.

The high-energy and high-intensity neutron source needs of programs other than for the CTR materials program were briefly described and outlined as:

1. Radiobiology and Medicine

2. Military Applications

3. Neutron Radiography (static and dynamic)

4. Solid State Science

5. Materials study for fast-fission reactors at high energies

6. Safety tests for fast-fission fuel elements. 
The extent to which the agencies involved in the above programs would participate in a source development project is presently unknown. However, they may be potential users and would broaden the base of support in operating such a facility. The levels of source intensity in each of the programs differ and in some cases may even exceed those levels usually associated with fusion reactors ( $210^{14}$ neutrons $/ \mathrm{cm}^{2}-\mathrm{sec}$ ). Therefore, the involvement by some of the agencies as users or participants will depend on the intensity level which can be obtained by the device.

The time-scale presented by $W$. Rice in the meeting (see Table I) appears in keeping with the overall expectation of developing a fusion reactor prototype. The emphasis on the technical assessment and preliminary conceptual design efforts in the next few years is reasonable and necessary. This phase of the study is necessary since the cost of the facility (regardless of concept) will range in the tens of millions of dollars. An initial investment in generating technical design data to support the feasibility of some of the concepts will be required to ensure that the facility eventually selected will have the maximum potential for successful development and maximum utilization.

The general impression arising from the discussion was initially the attitude that a plasma-driven neutron facility would not be different from the plasma confinement feasibility experiment. However, this changed markedly during the latter part of the morning session. This appeared to be based in part in delineating the differences in the objectives of the two facilities and consequent design requirements. Design requirements for a neutron facility would have to include a much higher duty cycle than a scientific feasibility experiment. However, the point to be made is that a neutron facility utilizing fusion devices would evolve and develop from the effort and developments in the plasma confinement program. A neutron facility would operate as a power consumer within economic constraints. Any combination of plasma injection and heating systems discussed at the meeting could be used in a neutronproducing reactor with efficiencies of these devices being of secondary importance. This would not necessarily be the case for scientific feasibility experiments. Defining the overall reactor efficiency $\varepsilon$, as the ratio of fusion power to input power (Okhawa), the general statements indicated that the efficiency can be such that a neutron source is feasible (Post on the mirror). To firm up these impressions will require further technical 
Table I

$14 \mathrm{MeV}$ Neutron Source Development

Activity

Assess Possible Approaches ( $\frac{1}{2}$ year)

Preliminary Source Design (1 year)

Supporting Research and Development (2 years)

Final Design \& Construction (Titles I-IV, 3 years)

Initial Operation and Debugging (1 year)

Irradiate Selected Materials \& Systems (4 years)

Feedback - Continuous

Testing of New Materials and Systems (4 years)

Data Assembly for Experimental Reactor Design (1 year)
Completion, FY

1973

1974

1976

1979

1980

1984

1988

1989

(Construction start for Mark II model about 1981 is Tikely) 
assessments involving a limited but selectively in-depth systems design study. The basic data would be developed from information generated by the on-going plasma confinement programs.

Without being too specific in the injection and heating systems described during the meeting, the impression was that in some instances cases were being made to present alternate techniques for neutron sources other than the two main fusion devices. Aside from this there seemed to be some merit in using a combination of these techniques in heating and forming plasmas to fusion conditions. The relativistic electron-beam and laser-beam heating devices may be developed as neutron sources but to scope the feasibility of these techniques would require the same level of technical scrutiny that has to be applied to all the concepts.

Of particular interest was that a neutron source utilizing the linear $\theta-$ pinch (high density pinch) technique was classed as a scientific feasibility experiment. It appears that to get neutron fluxes in the range of $10^{13}$ to $10^{14}$, a device approaching the Reference Theta Pinch Reactor is needed.

The mirror machine was briefly discussed in the context of using a superconducting system (baseball II). The parameters associated with this concept were more detailed than the others presented at the meeting as a consequence of having been subjected to a scoping study. Other possibilities such as the use of a modified and expanded version of a $2 X-I I$ device, or cryogenic magnets were not covered in the discussion.

There seemed to be some concern that a neutron flux of $10^{15} / \mathrm{cm}^{2}-\mathrm{sec}$ may be difficult to obtain assuming that this intensity would be adopted as a goal. This flux level was presented oniy as a starting point for the discussions. If the CTR materials program was the only program involved, this flux could be decreased. Flux levels in the range of $10^{14}$ may be acceptable. However, if development support from other programs is desired then the above source intensities may not be adequate.

Whether a plasma-driven neutron facility is possible and timely will depend on the progress made in the plasma confinement program. The success of the plasma confinement experiments, will be in evidence several years before the actual demonstration itself $(1980-1982)$. If in the next several 
years (1974-1977) the plasma confinement program produces evidence verifying the validity of the "scaling-laws," then the next most important major facility (other than the physics test reactor) in the development of fusion power is a fusion-neutron radiation test facility. Therefore, the decision to plan a neutron radiation facility can also be made in advance of the demonstration experiment.

The gas target concepts and dense plasma focus devices have been discussed in previous reports and its development potential should be made in comparison with other beam-target techniques such as the ICT rotating target, the drive-in target system and the self-replenishing tritium targets. These last two sys tems were reported at the Las Vegas Neutron Source Workshop meeting and were included in the summary of that meeting. The concepts are in the process of being developed and the progress of these efforts should be followed and evaluated.

One important aspect that must be introduced in the evaluation process for all concepts, is the engineering plan necessary to transform these devices into a useable and flexible research radiation facility. A most important phase of the design effort will be the scoping of a materials and engineeringtest experimental program. The experimental requirements of this program will be the major factor influencing the design feature of this facility. The accessibility of the neutrons to the experimenters; the cooling system requirenents for the reactor components and experimental apparatus; the design details to accommodate the materials and engineering test experiments; the maber, design and size of experimentál beam ports; the biological shield and penetrations; etc., all will impose constraints which will become major factors in the selection process of a concept. The time limitation of the meeting did not allow much discussion on this phase of the source design.

An attempt was made to summarize the possible approaches to a fusionreutron radiation facility, by tabulating some general specifications and cost estimates. The resulting Table II is attached. The utility of the information contained in the table has a high order of uncertainty, particularly in the capital cost column. The cost figures were not results from a studied approach to the concepts, so that capital costs and just as important operating costs of each system is at present unknown. 
TABLE II. POSSIBLE APPROACHES TO 14 MAV NEUTRON SOURCE

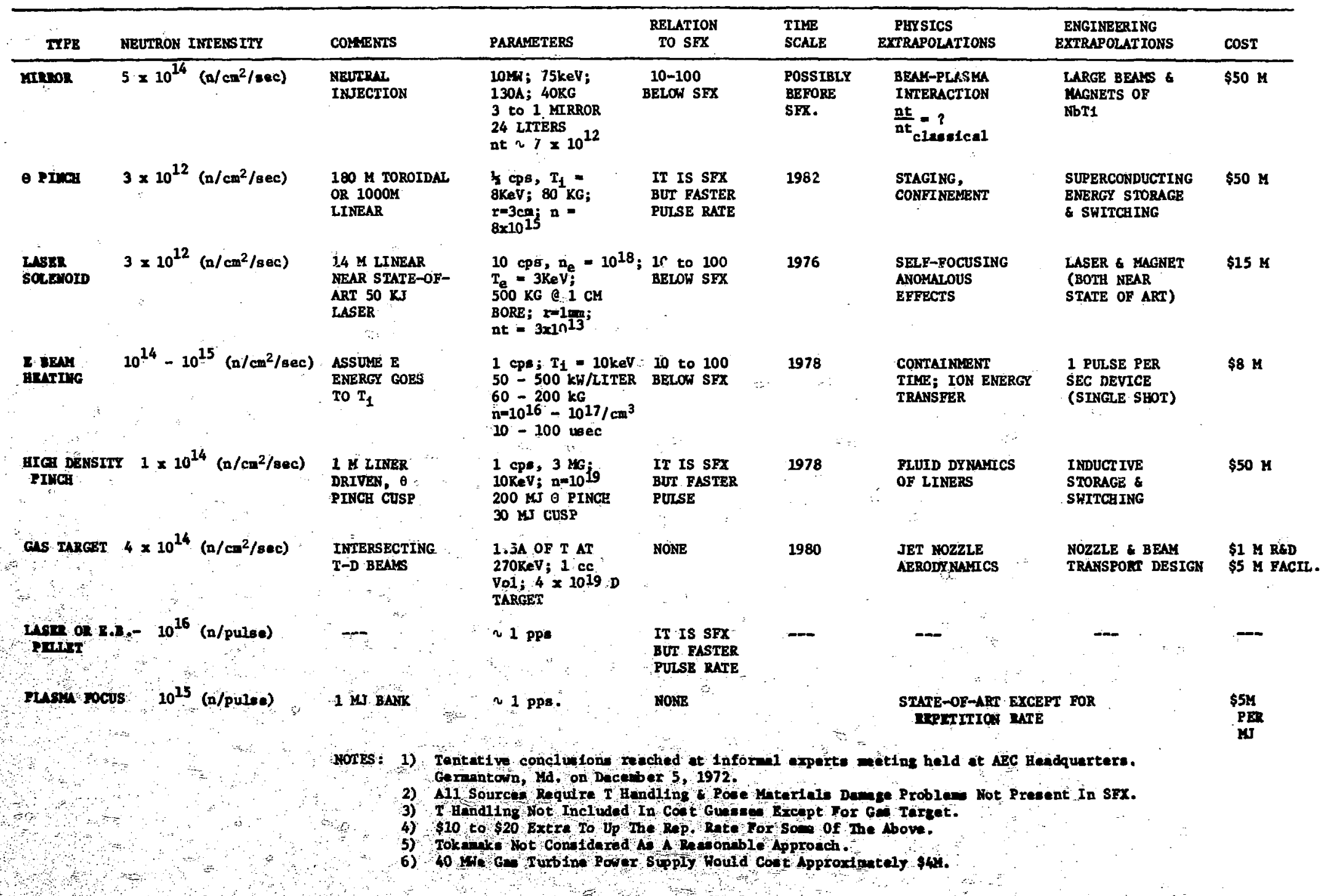


The capability and flexibility of a radiation test facility should be optimized in the design to allow several different experimental programs to be carried out simultaneously. This would require designing-in an adequate access capacity to the source region itself. Eventually the merits of a test reactor design must be based in part on the following considerations:

(a) the level of source intensity actually available to the investigator,

(b) the capability and flexibility of the source concept to accommodate a range of experimental needs,

(c) the number of experiments which can be performed simultaneously in order to provide a broad base of support for the total facility,

(d) the economics of the research and development necessary to establish the feasibility of a concept,

(e) the capital costs to build the facility, and

(f) the operating costs per experiment.

An objective of these meetings and discussions is to eventually outline an orderly approach to the development of a fusion-neutron radiation facility for the CTR Technology program. Since the development of these concepts necessarily involves great expenditures of effort and costs, the decision to build an intense neutron source must be based on a timely sequence of in-depth technical considerations. An attempt in this direction is made by introducing a classification system. The first-draft of this study, the Development of the Intense Neutron Source Facilities for the CTR Technology Program, is included in this report.

It is intended that this summary and documents can serve as the basis for planning the next level of discussions and effort in the development of irradiation test facilities for the CTR Technology program. 


\section{The Development of Intense Neutron Source} Facilities for the CTR Technology Program

\section{A. Introduction}

One of the major facilities necessary for the timely development of prototype and demonstration fusion-power reactor plants, is a materizis and engineering test fusion reactor. The primary function of this facility is to generate a radiation field environment (neutrons, photons, and energetic particles) for large-scale testing and engineering programs in areas such as: materials, blanket designs, superconducting systems design, instrumentation for reactor control, isotopic concentrations, liquid-metal coolant chemistry, heat transport mechanisms, mechanical designs, etc. The testing reactor and its corresponding engineering experimental program will be one of the main instruments in identifying and evaluating major problem areas, varying technical approaches, potential methods of solving design problems and establishing relative priorities in the research and development of fusion-power reactors. Some of the problem areas and neutron generating systems or concepts that would partially satisfy the requirements of the CTR materials program have been discussed by materials investigators and neutron source designers and are summarized in Ref. 1. Many devices such as solid and liquid targets, tritium drive-in targets, gas-targets, dense plasma focus devices laser-plasma devices, e-beam plasma devices as well as the magnetic field confinement devices have been considered as potential candidates for developing into intense neutron generators. The variety of these proposed facilities and concepts requires that a systematic program plan be established for the development of an optimum radiation and testing reactor directly related to the needs of the CTR Technology Program.

The purpose of this report is to initiate a basis for developing a program in-phase with the progress of the Plasma Confinement program and the long-range goal of constructing thermonuclear power plants.

\section{B. Classification of $(D, T)$ Neutron Facilities}

The variety of neutron source systems and the variety of the CTR programmatic needs necessitate introducing some order in classifying facilities

1. P. J. Persiani, "Neutron Source Considerations for the CTR Materials Program," Proc. of Workshop on High Intensity Neutron Generators, Las Vegas, Nevada, p. 111, June 1972. 
and concepts. This allows establishing a more realistic basis for assessment and evaluation within each category. In addition, it al lows the plan to be structured in a manner that eases assigning priorities for development, and just as important, introduces a degree of flexibility. The flexibility has two advantages:

1. Plan modifications can be made to phase-in with the general progress of development in the other CTR programs, and

2. Funding contingencies can be accommodated consistently with the assigned priorities.

The concepts are classified into five groups as outlined in this section. The three main groupings are based, in part, on the type of systems and the level of source intensity actually achieved or anticipated. The supplementary Group II-S is defined to indicate that the concept may be utilized as an auxiliary heating technique for other systems as well as a neutron source device. The Group III-S category consists of those fusion-plasma concepts associated with a somewhat more futuristic aspect to the state of the art.

\section{Classification of $(D, T)$ Neutron Facilities}

Group I - Accelerator and Target Systems

1. Rotating target sys tem

a. Solid $\mathrm{TiT}_{2}$ thin target

b. Sputtering-electrode $\mathrm{TiT}_{2}$ thick target

2. Drive-in target system

a. Mixed $(D, T)$ ion beam on solid $\mathrm{Cr}$ target

3. Self-replenishing target system

a. Tritium gas flow in solid $\mathrm{TiT}_{2}$ target

4. Liquid-jet LiT/Li mixture target

Group II

1. Dense plasma focus device

2. Supersonic gas-jet target

3. Liquid-jet target (LiT) 


\section{Classification of $(D, T)$ Meutron Facilities (cont'd)}

Group II-S

1. Laser-plasma interaction device

a. Auxiliary heating for Group III type concepts

b. Laser-plasma solenoid as neutron source

2. Relativistic electron beam-plasma interaction device

a. Auxiliary heating for Group III type concepts

b. Relativistic-electron beam-plasma as neutron source

3. Drift Orbit Cyclotron Heating (DOCH)

a. Auxiliary heating for Group III type concepts

Group III

1. Linear $\theta$-Pinch Machine

2. Mirror-Machine

3. Others

Group III-S

1. Laser-pellet interaction

2. Relativistic-electron-beam-pellet interaction

C. Design-Related Considerations

The general design features and problems and the anticipated contribution to the materials and engineering program for each of the five are reviewed and outlined in this section. The design-related considerations involve: the level of total neutron intensity or fluxes, source geometry, a statement on the effort required for the successful development of the concept, the relationship between the activities involved in developing the source and the activities involved in the CTR program, and an estimate of the range of costs in each of the categories.

Included in the outline are the anticipated contributions and the technical significance to the materials program from the experiments performed on the systems within each group.

The above general considerations are used as a guide in assigning priorities and funding levels in the plan for each of the concepts. 


\section{Type of $(D, T)$ Neutron Facility \\ Group I \\ Design-related Considerations}

1. Low intensity $>10^{13} \mathrm{n} / \mathrm{sec}$

2. Point geometry source

3. Limited experimental access

4. Low calculated risk in performance expectation

5. Modest scaling for accelerator and target development
6. State of art technology

7. Technology for source development not related to the main CTR development program in the SFX

8. Modest investment in $R$ \& $\$ 1,000,000$ to $\$ 1,500,000$

\section{Anticipated Contribution to Materials Program}

1. Initiate scoping experiments on effects of fusion-energy neutrons on materials

2. Observation of threshold or onset of damage phenomena

3. Assess significance of damage phenomena

4. Guidance in planning a materials development program directly relevant to fusion reactor systems

5. Contribute to establishing and planning specific output feature of the CTR Radiation Testing Facility

\section{Group II}

Design-related Considerations

1. Medium to high intensity $>10^{14} \mathrm{n} / \mathrm{sec}$

2. Point source geometry

3. Limited experimental access

4. Higher calculated risk in performance expectations than in Group I

5. Moderate to substantial scaling
6. Major $R \& D$ effort for development

7. Extending current technology

8. Technology for development peripherally related to main CTR program for SFX

9. Major Investment in $R \& D$ $>\$ 10,000,000$

\section{Anticipated Contribution to Materials Program}

1. Significant radiation fluence allowing study of materials damage over extended time periods

2. Improved assessment of damage phenomena (dimensional instability) for preliminary design studies

3. Neutron fluence effects on mechanical and physical properties for structural materials 


\section{Type of $(D, T)$ Neutron Facility \\ Group II-S \\ Design-related Considerations}

1. Medium intensity as a neutron source

2. Point, line, or extended source geometry

3. Alternate function to use as auxiliary heating in Group III and III-S

4. Higher calculated risk in performance expectation than Group I type
5. Substantial scaling required

6. Major $R \& D$ effort for development

7. Extending current technology

8. Technology for development marginally related to the main CTR program

9. Major investment in $R \& D$ $>\$ 15,000,000$

Anticipated Contribution to Materials Program

1. Indirectly as an auxiliary heating subsystem of a fusion neutron source

2. As a neutron source same as Group II systems

Gr

Group III

Design-related Considerations

1. Medium to high flux $>10^{14} \mathrm{n} / \mathrm{cm}^{2}-\mathrm{sec}$

2. Extended geometry

3. Extended accessibility to accommodate a range of experimental program

4. Lower calculated risk than "breakeven" thermonuclear machine

5. Substantial scaling required

6. Major $R \& D$ effort for development can be obtained from the main CTR SFX program
7. Extending current technology

8. Technology for development of a thermonuclear machine as a neutron source is related directly with the main CTR program

9. Major investment in $R \& D$ $>\$ 30,000,000$

Added note: The current SFX $R \& D$ program forms the bas is for development of the intense fusion neutron radiation facility

Anticipated Contribution to Materials Program

1. Materials and engineering testing program in environment characteristic of a fusion power reactor

2. Testing program in this environment include:
a. materials damage
b. blanket designs
c. superconducting systems design
d. instrumentation for reactor control
e. isotopic concentrations
f. Tiquid-metal coolant chemistry
g. heat transport mechanisms
$h$. mechanical designs
i. etc. 


\section{Type of $(D, T)$ Neutron Facility}

\section{Group III-S}

Design-related Considerations

1. Medium to high flux $>10^{14} \mathrm{n} / \mathrm{cm}^{2}$-sec

2. Extended geometry

3. Extended accessibility to accommodate a range of experimental programs

4. Lower calculated risk than "breakeven" thermonuclear machine

5. Substantial scaling required
6. Major $R \& D$ effort for development

7. Long-range technology and development

8. Technology for deve lopment related directly with laserinduced fusion CTR program

9. Major investment in $R \& D$ $>\$ 30,000,000$

Anticipated Contribution to Materials Program

1. Materials and engineering testing program in environment characteristic of a laser induced fusion power reactur 


\section{Major Research and Development Areas}

\section{1 Introduction}

The purpose of this section is to identify and assess some of the design problems involved in the various types of existing neutron facilities or concepts which can be utilized in the CTR technology program. The R \& D problem areas are presented only as a starting point and as a guide for a more timely and in-depth evaluation of the facilities as the designs progress.

The overview at this point is necessary in order to put into better perspective the balance between the magnitude of the effort required to successfully develop a system and the user's conviction and assurance that the output of the facility is just the quality output required to make major technically significant advances directly relevant to the CTR technology program.

\section{D.2 Subsystem Deve lopment Areas Common to Several Types}

There are two major development areas which are common to most of the concepts listed; a) radiation effects on materials; and b) pulsed energy storage and transfer system.

\section{D.2.a Radiation Effects of Materials}

The development of structural materials to withstand neutron, charged particle and photon radiation fields will be of major concern in the design of any fusion facility. The major $R \& D$ effort in materials for the radiation facility will be in cormon with, if not identical to, the program for fusion power reactors. The difference is that the high-fluence design constraints usually associated with a power reactor can be considerably relaxed for a test reactor. The reason is that the frequency of structural component replacement in the test facility will be influenced mostly by the programmatic needs and can be integrated into the design features. The replacement frequency for the power reactor components will be strongly influenced by the systems economics and physical design constraints imposed by interfacing subsystems.

The materials development for a neutron source facility would have to evolve from data generated from experiments performed on the Group I type facilities as well as on ion-bombardment simulation experiments, existing accelerator-Be target systems, and experience in fast-fission spectra. 
The design parameters would be based on extrapolations from these data to the conditions expected in a neutron source facility. The extrapolation could be effected with a good degree of confidence. Depending on the specific facility design, the structural components need not be subjected to the severe radiation environment anticipated in a high-power-density fusion reactor.

\section{D.2.b Pulsed Energy Storage and Transfer System}

Several of the $(D, T)$ neutron source concepts are essentially

pulsed systems. The major problem in this area is the energy storage and transfer system. The feasilibity of developing a neutron facility in a timely and meaningful manner to the overall CTR program is critically dependent on the approach taken for the energy storage system. For storage systems in the range of several megajoules, conventional capacitor bank systems with conventional energy switching ignitrons appear capable of being developed with repitition rates in the order of several pulses per second. This eases the necessity of having to depend primarily on the development of the superconducting magnetic energy and traisser system.

A modest development program to produce comercially available capacitors and switching systems having a lifetime of $10^{7}$ cycles would not introduce a time-delay in the utilization of some of the proposed pulsed neutron facilities.

In the remainder of this section, the preliminary identification of limitations and problems are outlined for each type of facility or concept within each group. Also included in the tabulation is a listing of the $R$ \& $D$ effort necessary to translate each concept into a potential neutron source for use in the CTR program. 
Limitations and R \& D Effort

Group I

1a. Solid $\mathrm{TiT}_{2}$ thin target

Limitations

R \& D Effort

1. Beam power dissipation in target 1. Increase target size

2. Low neutron yield $\sim 2$ to $3 \times 10^{12} \quad$ 2. Increase ion-beam current from $\mathrm{n} / \mathrm{sec}$ 1.0 $\mathrm{mA}$ to $24 \mathrm{~mA}$

3. Limited target half-life $\sim 700 \mathrm{mAh}$ 3. Tritium handling and recovery system Expected improvement: 1. Neutron yield $\sim 4$ to $6 \times 10^{12} \mathrm{n} / \mathrm{sec}$

lb. Sputtering-electrode $\mathrm{TiT}_{2}$ thick target

Limitations

1. Technique being developed only for $\mathrm{TiD}_{2}$ thick target

2. Testing at low beam-currents (1.5 $\mathrm{mA})$ and beam-energies (100 keV)

3. Unknown flux attainable
R \& D Effort

1. Apply technique to $\mathrm{TiT}_{2}$

2. Handling of $T_{2}$ emitted during sputtering

3. Attain beam-current $30 \mathrm{~mA}$ and beam-energy $300 \mathrm{keV}$

Expected improvements: 1. Neutron yield $\sim 10^{13} \mathrm{n} / \mathrm{sec}$

2. Target half-life $>9000 \mathrm{mAh}$

2. Mixed $(D, T)$ ion beam incident on $\mathrm{Cr}$ target

Limitations

1. Limited $(D, D)$ neutron intensity $24 \times 10^{10} \mathrm{n} / \mathrm{sec}$

2. Beam area $\sim 13 \mathrm{~cm}^{2}$

3. Not a point source geometry

4. Low close-in flux
R \& D Effort

1. Develop techniques for $(D, T)$ beam

2. Improve beam focusing to much smaller diameter

3. Attain beam-currents $\sim 250 \mathrm{~mA}$

4. Attain beam-energy $\sim 300 \mathrm{keV}$

5. Tritium handling and recovery system

Expected improvements: 1 . (D,T) neutron intensity $4 \times 10^{12} \mathrm{n} / \mathrm{sec}$

2. Unlimited target lifetime 
Limitations and R \& D Effort

Group I

3. Self-replenishing tritium target

Limitations

1. Technique developed for low beam currents (1 mA)

2. Target yieid of $2 \times 10^{10} \mathrm{n} / \mathrm{sec}$ sustained for $10 \mathrm{hrs}$.
R \& D Effort

1. Extend technique to beam currents ఒ30 $\mathrm{mA}$ or higher

2. Tritium gas handling and recovery system

3. Develop cooling system for greater beam-power and sustained lifetime

Expected improvements: 1. Higher $(D, T)$ neutron yield at higher current 2. Extended target lifetime

4. Liquid-jet (LiT/Li mixture $\leq 1 \%)$

Limitations

1. Initial conceptual stage

2. Tritium availability
R \& D Effort

1. Heat removal for liquidous

(LiT/Li) systems

2. Compatibility and coolant chemistry

3. Tritium inventory

Expected improvements: 1. (D,T) neutron yields $>10^{13} \mathrm{n} / \mathrm{sec}$

2. Ease of tritium control because of being chemically bound

3. $R$ \& D eifort on $L i T / L i$ system relates directly to CTR program 
Limitations and R \& D Effort

Groun II

1. Dense Plasma Focus

Limitations

1. Scaling neutron yield with energy

2. Attained $(D, T)$ neutron yield $6 \times 10^{12} \mathrm{n} / \mathrm{sec}$

3. Energy storage system for energies $>$ several MJ

4. Electrode lifetime at high repetitive rate (radiation damage)

5. Limited access to neutron source region

6. Tritium contamination

Expected improvements: 1. Increase $(D, T)$ neutron yields to $210^{15} \mathrm{n} / \mathrm{sec}$ depending on system energy

2. Neutron yield increase directly proportional to repetitive rate
R \& D Effort

1. Establish $(D, T)$ neutron yieldenergy scaling laws (MJ range)

2. Engineering heat-dissipation system for electrodes and energy storage bank

3. Tritium handling and recovery system

4. Engineering safeguards for tritium enviroment

\section{Supersonic gas-jet target}

\section{Limitations}

1. Conceptual stage

2. Stability of ion beam currert density of $>1 \mathrm{~A} / \mathrm{cm}^{2}$

3. Focusing beam into $<1 \mathrm{~cm}^{2}$ nozzle area

4. Heat dissipation in jet nozzle region

5. Effect of heat load on aerodynamics of supersonic jet-flow

6. Jet nozzle design integrity and lifetime (radiation damage)

7. Tritium contamination

8. Limited access to neutron source region 


\section{Limitations and R \& D Effort}

\section{Group II}

3. Liquid-jet target (LiT/Li mixture $>1 \%$ )

\section{Limitations}

1. Initial conceptual stage

2. Tritium availability
R \& D Effort

1. Heat removal for liquid (LiT, Li) systems

2. Compatibility and coolant chemistry

3. Tritium inventory system

Expected improvements: $1 .(D, T)$ neutron yields $>10^{14} \mathrm{n} / \mathrm{sec}$

2. Ease of tritium control because of being chemically bound

3. $R \& D$ effort on $L i T$, Li system relates directly to CTR program 
Limitations and R \& D Effort

\author{
Group II-S \\ 1. Laser-Plasma Interaction Device \\ a. Auxiliary Heating
}

Limitations

1. Initial conceptual stage

2. Mostly theoretical model

3. Limi ted experimental data

4. Uncertain energy coupling between

laser-plasma for heating ions
R \& D Effort

1. Experimental data on heating mechanism

2. Follow development in laserplasma interaction program

b. Laser-Plasma Solenoid Neutron Source

Limitations

1. As above

2. Plasma confinement magnetic fields
R \& D Effort

1. Follow development in laser-plasma interaction and confinement program

2. Plasma confinement progran

Expected improvement: 1. Auxiliary heating technique for Group III type neutron facility

2. Relativistic-Electron Beam-Plasme Interaction Device a. Auxi liary Heating

Limitations

1. Initial conceptual stage

2. Mostly theoretical model

3. Limited experimenta data

4. Uncertain energy coupling between electron beam and plasma for heating ions although initial experiments indicate coupling between bean electrons and plasma electrons
R \& D Effort

1. Adequate scaling for utilizing technique

2. Experimental data on heating mechanism

3. Follow development in electron-beam and plasma interaction program 
Limitations and $R$ \& $D$ Effort

Group II-S

2. Relativistic-Electron Beam-Plasma Interaction Device

b. E-Beam-Plasma Solenoid Neutron Source

Limitations

1. Initial conceptual stage

2. Current yield estimate $10^{14}-10^{15}$ $\mathrm{n} / \mathrm{pulse}$ in $4 \mathrm{M}$ distance
R \& D Effort

1. Follow development in electronplasma interaction and confinement program

2. Plasma confinement program

Expected improvement: 1. Auxiliary heating technique for Group III type neutron facility

3. Drift Orbit Cyclotron Heating (DOCH)

Limitations

1. Initial conceptual stage

2. Mostly theoretical model

\section{R \& D Effort}

1. Experimental verification

2. Follow development in mirror machine auxiliary heating systems

Expected improvements: 1. Auxiliary heating technique for Group III type neutron facility 


\section{Limitations and R \& D Effort \\ Group III}

\section{Linear $\theta$-Pinch Machine}

\section{Limitations}

1. Absence of conceptual plan for development as a neutron facility

2. Plasma temperatures, density and confinement times as experienced so far in the CTR program

3. $<10^{14} \mathrm{n} / \mathrm{cm}^{2}-\mathrm{sec}$ is estimated for the SFX

\section{Mirror Machine}

\section{Limitations}

1. Limited conceptual stage based on Basebal 1-II

2. Absence of conceptual plan for development of $2 X-I I$ as a neutron facility

3. Plasma temperatures, density and confinement times as experienced so far in the CTR program Neutron Radiation Facility (INRAF) for the long range CTR program

R \& D Effort

1. Initiate conceptual design for developing the linear $\theta$-pinch as a neutron facility

2. Plasma density, confinement and temperature conditions as developed in the CTR-SFX program

3. Follow experimental developments in auxiliary heating device and convert into design concepts

4. Utilize the experimental development in all aspects of CTR related programs into design concepts for neutron source

Expected improvements: 1. Engineering layout of an extended Intense 
Limitations and $R \& D$ Effort

Group III-S

1. Laser-Pellet interaction

Limitations

1. Absence of conceptual plan for development as a neutron facility

2. Developments in laser program

3. Experimental data relating to laser-pellet energy transport
R \& D Effort

1. Timely initiation of conceptual design for developing as a neutron facility

2. Follow developments in the laserpellet interaction program

3. Follow development of high-power lasers

2. Relativistic-Electron Beam-Pellet interaction

Limitations

1. Absence of conceptual plan for development as a neutron facility

2. Development in high-current field emission electron beams

3. Electron beam-pellet energy transport
R \& D Effort

1. Timely initiation of conceptual design for developing as a neutron facility

2. Follow developments in the general field of relativistic-electron beam-pellet interaction program 


\section{E. Further Considerations on Source Facilities}

\section{E.1 Thermonuclear-Radiation Facility (Group III and III-S)}

The success of the scientific plasma confinement experiments will be in evidence several years before the actual demonstration itself (1981-1982). If in the next several years (1974-1977) the plasma confinement program produces evidence verifying the validity of "scaling-laws," then the next most important large scale facility (other than the plasma physics test reactor) in the development of fusion power is a plasma-driven neutron radiation facility. Once a high degree of assurance is established that adequate plasma densities, temperatures, and confinement times are attainable, then the thermonuclear plasma principle should be the basis of design for the materials and engineering test reactor.

The system would not need to be a net-power-producing reactor. Therefore many of the severe engineering design constraints usually associated with power-producing fusion reactors would be substantially reduced or relieved. For example: non-breeding blankets; water, gas, or sodium coolants; stainless steel first wall; lower wall temperatures; a variety of plasma heating and injection systems; superconducting, non-superconducting or cryogenically cooled magnet coils; etc. The mirror and the linear thetapinch have been suggested for the initial study phase since these devices appear to involve physical dimensions much smaller than toroidal systems. However, the possibility of tokamaks or laser-induced fusion devices as neutron scurces must always be allowed for in any plan.

A thermonuclear plasma device has the advantage of having an extended source of neutrons. The geometrical dimensions of the source can be selected with reasonable flexibility to accommodate, simultaneously, a variety of experiments. A numbei of horizontal and vertical experimental beam holes would provide close-access to regions of high neutron fluxes. The merits of the concept must be based in part on the following considerations:

(a) the level of source intensity actually available to the investigator;

(b) the capability and flexibility of the source concept to accommodate a range of experimental needs; 
(c) the number of experiments which can be performed simultaneously in order to provide a broad base of support for the total facility; and

(d) the economics of the research and development necessary to establish the feasibility of a concept, the capital costs to build the facility, and the operating cost per experiment.

Another advantage is that the $R$ \& $[$ effort would closely relate and actually be consistent with the general CTR plasma confinement research and the design problems involved in the development of fusion reactors to demonstrate scientific feasibility. In fact the main design effort for the neutron facility would not be a completely separate effort. It would follow and utilize the developments in the SFX effort and translate the developments into the design of the radiation facility.

A materials-testing fusion reactor would provide a neutronic environment characteristic of a fusion power reactor. The testing program in this environment would include: materials, blanket designs, superconducting systems design, instrumentation for reactor control, isotopic concentrations, liquid-metal coolant chemistry, heat transport mechanisms, mechanical designs, etc. The role that this facility would play in the development of a prototype or demonstration plant would be analogous to the role played by the materials testing and experimental reactors in the development of fission power reactors.

\section{E.2 Group II and Group II-S Concepts}

Attempts to develop a neutron source representative of Group II and II-S devices appear to require a substantial $R \& D$ program. The design effort for certain phases of the Group II devices would be a marked diversion from and dilution of the resources for the main thrust of the plasma physics program. The timely development of a neutron source system should be influenced by and in-phase with the progress in the confinement program.

The investment in effort should be commensurate with the state of development in the SFX program, since it is only on this basis that a substantial and long-range materials program can be justified. A time-differential of a few years may be gained between developing Group II and Group III type systens. However, this gain in time-differential must be put into a balanced perspective with respect to: 
a. the time-needs of the overall CTR program,

b. the magnitude of the effort invested and the expected technically-significant output,

c. the lifetime of the system in contributing to the overall program,

d. the long-range needs of the materials program,

e. the need and time to initiate the development of the ultimate radiation facility to satisfy the long-range technology program,

f. the gains attained if the magnitude of the effort has been initially directed to the thermonuclear radiation facility.

\section{E.3 Target Development (Group I)}

In order to partially meet the near-term needs of the technology program, a modest effort in development of target and increased ion-beam current systems should be considered. With limited funding in the early years for source development, the technical data requirements in the conceptual design-phase studies for fusion power reactors should be stressed. The designers will require neutron-related data having "a good degree of reliability" for extrapolations to the design conditions of a power reactor. The uncertainties in the design parameters should be kept reasonably minimal. The source requirements for this phase of the technology program seems to indicate that emphasis be given to upgrading the intensity of existing systems in the Group I category. This specifically $(D, T)$ neutron data can also be supplemented by experiments on existing accelerator-Be target systens (20-50 MeV deuteron energies and 10-150 $\mathrm{AA}$ currents), ion-bombardment simulation experiments, and experience in fast-reactor spectra. The magnitude of the effort should be consistent with the projected needs in the next 4 or 5 years, and the ability to make available $(D, T)$ neutron intensities in the range of $10^{13}$ to $10^{14} \mathrm{n} / \mathrm{sec}$ (or $210^{13} \mathrm{n} / \mathrm{cm}^{2}-\mathrm{sec}$ ).

\section{F. Summary}

In balance, therefore, the feasibility of utilizing any of the fusionreactor concepts as an intense neutron radiation facility should remain high in the order of priorities.

A possible scheduling of effort to effect a timely assessment and intercomparison of source concepts, which is consistent and in-phase with the progress of the plasma confinement program is included in Table III. As 
Table III

Scheduling

Plasma Confinement Program

and

Radiation Test Facility Program

\begin{tabular}{|l|c|c|c|c|c|c|c|c|c|c|}
\hline CY & 1973 & 1974 & 1975 & 1976 & 1977 & 1978 & 1979 & 1980 \\
\hline FY & 1974 & 1975 & 1976 & 1977 & 1978 & 1979 & 1980 \\
\hline
\end{tabular}

\section{Radiation Test}

Facility Programs

Group I

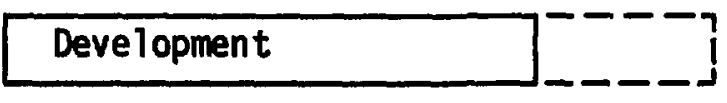

Group II

$$
\text { Concept Design Studies }
$$

Group III

$$
\text { Concept Design Studies }
$$

Design

Princeton

Large Torus

PLT

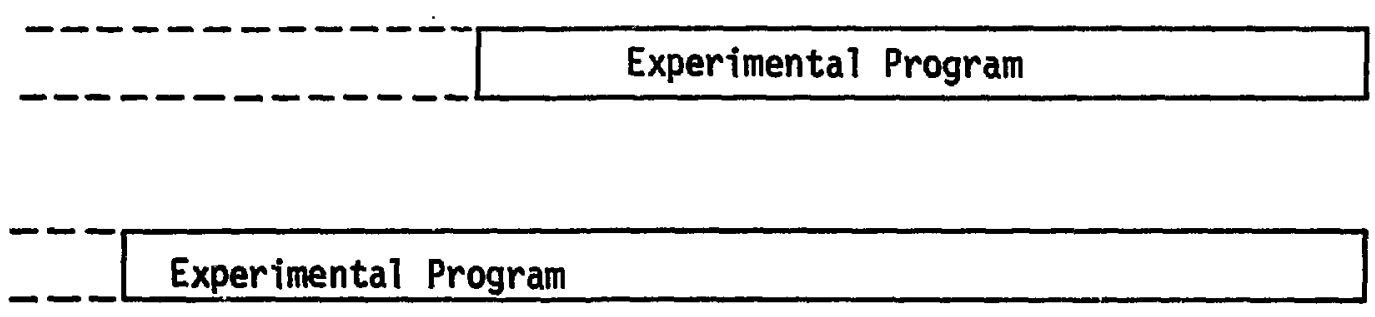

LASL

SCYLLAC

Experimental Program

LLL

MIRROR

Experimental Program 
discussed in the preceding section, the efforts defined in FY 1974 to FY 1976 wi 11 be most critical in the formulation of an optimum long-range (10-15 years) plan for the development of a major Radiation Materials Test facility, if the need is established by the successful progress in the confinement program. The initial experimental results from the plasma physics program are expected sometime in or by FY 1976. This allows a two or three year period to assess and to technically and economically evaluate neutron source concepts which can be transformed into an effective test facility or facilities to satisfy the broad class as well as the variety of needs of the materials and engineering test experiments.

A most important and initial phase of this effort should be the formulation of an overall materials testing program. The identification and articulating of the specific needs of this broad materials, surface phenomenon and engineering test program will be the major factor in influencing the selection and design features of the optimum test facility.

A start in this direction may be to form a CTR Neutron Source Group. The group would be a working-design-group comprised of experts in the relevant CTR laboratories and institutions. The collective and individual expertise of these organizations can be coordinated in this group effort. The designgroup would have the responsibility to plan, design and coordinate the overall effort in developing the neutron radiation facilities for the CTR program. It would be responsive to the needs, guidance and policy of the AEC CTR division. The responsibilities of the Design Group would include:

a. Identifying, evaluating and coordinating the interface problem areas of various disciplines involved in the design and the users' needs into a studied effort toward the goal of developing a radiation facility,

b. Formulating the materials experimental program which will influence the design features,

c. Initiating and developing new areas of effort necessary to resolve problems within each of the disciplines as well as across the disciplines,

d. Evaluating the technical significance of developments and progress in other CTR programs and translating these into the design of the facility, 